\title{
Gestión y cultura escolar. Referentes analíticos alrededor de la reforma educativa $^{1^{*}}$
}

\author{
Management and School Culture. Analytical References About Educational Reform
}

Recibido 23 mayo 2013 • Aceptado 26 junio 2013 • Corregido 26 junio 2013

\begin{abstract}
Resumen. El presente artículo discute algunos puntos de articulación entre la gestión y la cultura escolar como una vía necesaria para avanzar en la comprensión del funcionamiento real de las escuelas en contextos contrastantes y complejos. En tal perspectiva, se reconoce que al interior de los centros existen códigos culturales configurados históricamente, los cuales mantienen una relación dialéctica con la instrumentación de una política, un programa o un plan de carácter emergente orientado a la mejora de los procesos. La gestión y la cultura escolar, por tanto, constituyen elementos centrales de atención para indagar los diferentes comportamientos asociados a los imperativos actualmente en curso referidos a la calidad, la innovación, la eficiencia; los cuales influyen en las prácticas de quienes tienen la responsabilidad de llevar a cabo los compromisos emergentes de la educación.
\end{abstract}

Palabras clave. Gestión, cultura, organización, escuela.

\footnotetext{
* El presente artículo forma parte de los trabajos realizados en el Seminario "Gestión y cultura escolar" que incluye a estudiantes y académicos de la Universidad Pedagógica Nacional Unidad 098, en México D.F. Agradezco a los integrantes de dicho seminario la oportunidad de hacer públicas las siguientes reflexiones; sobra decir que las ideas expresadas son responsabilidad exclusiva de su autor.

2 Doctor en Estudios Sociales por la Universidad Autónoma Metropolitana, plantel Iztapalapa, México, D.F. Catedrático de la Facultad de Estudios Superiores Aragón de la Universidad Nacional Autónoma de México y académico de tiempo completo en la Universidad Pedagógica Nacional, Unidad 098 en México, D.F. Es coordinador de la especialización en "Gestión y Procesos Organizacionales en Educación Básica" dentro del Programa de la Maestría en Educación Básica de la Unidad UPN, 098. Actualmente coordina el proyecto de investigación "Gestión y cultura escolar. Referentes de significación alrededor del ejercicio docente" con la colaboración de académicos y alumnos de maestría de la Unidad UPN 098. Ha impartido cursos de actualización docente en distintos estados de México relacionados con la operación del Programa de la Maestría en Educación Básica. Sus últimas publicaciones son: 1) "Desigualdad, mercado laboral y educación superior en América Latina” para la Revista El Cotidiano, No. 176, 2012; 2) “Moda y trabajo: la expresión sociocultural de un saber hacer" para la Revista Nueva Antropología, Vol. XXIV, Núm. $75,2011 ; 3)$ "Política pública y sindicalismo magisterial: la búsqueda de la calidad educativa y los nuevos actores" para la Revista El Cotidiano, No. 168, 2011.
} 


\begin{abstract}
This paper discusses some points of articulation between the management and the school culture as a path to advance in understanding of the real operation of the schools in contrasting and complex contexts. In this perspective, it is recognized that inside the centers are configured historically cultural codes, which maintain a dialectical relationship with the instrumentation of a policy, program or a plan of emerging nature oriented to the improvement of processes. The management and the school culture, therefore, form a central element of attention to investigate the different behaviors associated to the imperatives currently in progress relating to the quality, innovation, efficiency; which influence the practices of those who have the responsibility to carry out the emerging commitments of the education.
\end{abstract}

Keywords. Management, culture, organization, school.

\title{
Introducción
}

A lo largo de estas tres últimas décadas, los países de América Latina han incorporado procesos de reforma educativa a nivel básico en distintos grados y con alcances muy diversos. Un aspecto central en estas propuestas lo constituye el replanteamiento de los principios de la organización escolar y, por extensión, del tipo de comportamiento desplegado por sus respectivos agentes.

Desde este referente, la gestión escolar se asocia fuertemente con la idea de cambio educativo por cuanto implica la instrumentación de un marco de acción alejado del paradigma burocrático caracterizado por la verticalidad de las decisiones, la estricta división del trabajo, la estandarización de los roles, la marcada separación entre lo administrativo y lo pedagógico, así como el descuido del aprendizaje como eje articulador de la educación. De este modo, la inclusión de categorías como <<eficacia $>>$, $<<$ calidad $>>$, <<liderazgo $>>,<<$ competencia $>>$; han dado pie a discusiones amplias sobre su significado en el terreno de la enseñanza, junto con su posible articulación con las finalidades sustantivas de la educación (Aguerrondo, 1993; Díaz Barriga, 2006; Hargreaves y Fink, 2008).

A la luz de esta nueva racionalidad conferida a lo educativo, resulta necesario recuperar la importancia del análisis cultural que abreve la discusión sobre los alcances de la gestión que se trata de impulsar en los centros de educación básica. ${ }^{3}$ Esto por cuanto los nuevos requerimientos educativos poseen implicaciones culturales en razón de incorporar una resignificación de las

3 La incorporación del término <<gestión>> en la jerga educativa ha venido progresivamente a sustituir la noción de <<administración escolar >>. No obstante, esto no sugiere que dentro del ámbito educativo se haya descubierto "de repente" el término gestión, como si fuese un concepto de nuevo cuño; siendo que ha estado presente desde hace mucho tiempo en la ciencia política y en las teorías de la organización, particularmente. Su singular uso en esta esfera responde a una resignificación de la organización escolar en el marco de las reformas educativas implementadas desde los años ochenta del siglo pasado. Ello como parte de la necesidad de instaurar mecanismos de autogestión en línea directa con la descentralización del servicio público, y que definiera el sentido de la reforma del Estado dentro de los países latinoamericanos en los primeros años del neoliberalismo. 
disposiciones, las prácticas, las tomas de decisión, los mecanismos de participación, así como las formas de gobernar y conducir las escuelas. En tal perspectiva, el análisis del funcionamiento de los centros pasa necesariamente por la recuperación de la dimensión cultural por ser un componente clave que explica las formas de organización social configuradas en torno a determinados propósitos educativos. Con base en este interés, el presente trabajo se aboca a remarcar las vías de articulación analítica entre gestión y cultura escolar, en tanto elementos fundamentales para la conformación de una agenda de investigación entrado el nuevo siglo.

\section{La gestión y sus vínculos con la cultura}

Pensada como organización, la escuela constituye un escenario bajo el cual se establecen formas de comportamiento singulares entre sus distintos participantes alrededor de una intencionalidad educativa. Esta última, por lo general, se traza con base en determinados ordenamientos institucionales que le confieren un carácter prescriptivo y regulador al trabajo cotidiano de maestros, alumnos, directivos y demás miembros organizados en torno a la labor pedagógica. Dentro de este ámbito, las lógicas de acción vienen orientadas por un esquema organizacional que ordena la disposición de los usos del tiempo y del espacio, de los recursos, de la división del trabajo, de las formas de comunicación, de las relaciones jerárquicas así como de la infraestructura y equipamiento para garantizar las actividades asociadas con el aprendizaje de los estudiantes.

Por extensión, el funcionamiento de los establecimientos escolares no está desprovisto de influencias por parte del entorno; por el contrario, constituye un modo de operación en el que se imbrican procesos sociales de diversa magnitud, cuyo origen rebasa frecuentemente los límites organizativos del quehacer educativo.

En consecuencia, al interior de la escuela, secombinan racionalidades propias dela actividad escolar con elementos del contexto social y cultural que le confieren un carácter particular a las interacciones cotidianas desarrolladas por los sujetos (Rockwell, 2005). Esto es posible por cuanto los centros no son entidades autónomas con capacidad de autorreproducción; fungen más bien como instancias de producción e intercambio simbólico con diversas franjas de la vida social. Dentro de este proceso, entran en relación variados órdenes de significación alrededor de lo educativo, cuyas implicaciones en el terreno de la enseñanza propician la recuperación de contenidos diversos y contrastantes acerca de lo útil o valioso del conocimiento transmitido.

Esta consideración plantea la necesidad de examinar los procesos de codificación de los dominios culturales que estructuran una visión educativa dentro de un particular contexto histórico. Sobre la base de estos dominios se instituyen esquemas colectivos que dan pie a la conformación de prácticas docentes, estilos de enseñanza, modos de interpretar y operar el currículo, formas de relacionarse con los alumnos, juicios de valor sobre el alcance de los 
aprendizajes, así como distintas convenciones sobre cómo organizar las actividades escolares; todo ello como parte de un entramado de significados con diferentes grados de arraigo o consolidación sobre los que opera un específico campo de saberes. La presencia de estos esquemas, junto con sus manifestaciones prácticas, adquiere tal poder que llega a "actuar como una prevención contra los modelos de innovación voluntaristas existentes en educación que descuidan, de manera frecuente, el elemento humano y cultural enraizado en el quehacer educativo" (Sacristán, 1999, p.106).

\section{Apropiación y memoria: su relevancia analítica}

Desde el campo antropológico, se han presentado en los últimos años aportaciones importantes para el estudio de la cultura escolar dentro del contexto latinoamericano. De manera especial, las aproximaciones ofrecidas por Rockwell (2005) han tenido un impacto significativo. Parte de su contribución reside en incorporar una categoría analítica clave para el tratamiento de estos procesos; a saber: <<la apropiación>>, entendida como aquella acción por la cual los sujetos toman posesión de los recursos culturales disponibles para organizar sus prácticas dentro de espacios de interacción concretos. 4

En el terreno de la enseñanza, la apropiación permite recuperar las formas culturales brindadas por el contexto junto con sus respectivos entendimientos al momento de emprender una acción educativa. Dicha perspectiva requiere una historia social de los usos discursivos así como de los modelos pedagógicos o educativos que poseen resortes de orden cultural. A partir de esta postura, se puede decir que la cultura no es simplemente un elemento accesorio o referencial para dar cuenta del funcionamiento de los centros; constituye más bien el eje estructurador por el cual se configuran sistemas de pensamiento y formas de conducción que ocurren al interior del centro escolar (Rivas, 2003).

En tal sentido, el estudio sobre la gestión no puede restringirse a la simple identificación de sus grados de correspondencia o adecuación con respecto a los imperativos funcionales desprendidos de las políticas educativas; se requiere antes bien analizar los procesos por los cuales se construye históricamente un tipo de organización que produce, incorpora y recrea determinados códigos culturales alrededor de la socialización educativa; y cuyas implicaciones en la forma de asumir un sentido de la educación pueden dar paso a modos de actuación diversos y contradictorios.

4 Dicha categoría es retomada del historiador francés Roger Chartier, para quien la apropiación implica metodológicamente atraer la historia social de los usos e interpretaciones de sentido que le dan las personas a diversas situaciones dentro de su vida en sociedad (Krauss, 1999). 
Esta condición exige analíticamente entender a la escuela, ante todo, como un grupo social. De ello se deriva que la apropiación es un ejercicio inserto dentro de complejas relaciones sociales a través de las cuales se ponen en juego conocimientos, intereses, expectativas o propósitos que pretenden ordenar las voluntades colectivas más allá de las preferencias individuales. En este proceso, los principios educativos no actúan como elementos incontrovertibles o suficientes por sí mismos, sino como órdenes de significación susceptibles de negociación, resistencia o consenso entre los distintos participantes. Es a partir de las <<interacciones culturales >> entabladas por estos agentes como la organización del trabajo educativo se liga a una variedad de tradiciones docentes, cuyos contenidos son el resultado de un acervo simbólico construido históricamente.

En ese nivel, resulta oportuna la recuperación de la $<<$ memoria $>>$ como una categoría adicional que nos puede auxiliar en la comprensión de las realidades escolares desde un sentido cultural. Aquí la memoria debe ser entendida como proceso social, es decir, como parte de una recuperación e invención de tradiciones compartidas por un conjunto de individuos desde un particular contexto. Es lo que para autores como Halbwachs (1968) o Candau (2002) constituye el $<<$ marco colectivo de la memoria $>>$, de naturaleza distinta a la memoria individual, por cuanto supone una representación colectiva del pasado en el que conviven a un tiempo la permanencia y la reconstrucción de sentidos asociados a eventos o acontecimientos diversos, cuyo impacto o significación se evalúa a partir de la cultura edificada por los sujetos a lo largo del tiempo. Es a través de la apropiación y la memoria como estos últimos hacen uso de diversos recursos culturales para organizar sus prácticas y sustentar un principio de identidad5 en el seno de una organización.

\section{La escuela como una trama cultural}

La comprensión de las acciones destinadas a la mejora de la escuela no puede desprenderse de los significados que tienen para las personas adecuar los valores y las actitudes a los nuevos requerimientos de la gestión. Ello plantea remarcar la importancia de las construcciones de sentido alrededor de esquemas centrados en la horizontalidad de las decisiones, el trabajo en equipo, el liderazgo compartido, la evaluación del desempeño docente, la participación comunitaria, etc. que suponen una ruptura con el modelo centralista y burocrático. Mediante esta vía se pueden identificar los procesos de articulación entre las experiencias cotidianas con los contenidos emergentes acerca de la educación y sus efectos pedagógicos concurrentes.

\footnotetext{
5 Al respecto, Giménez (2009) advierte que identidad y cultura poseen una relación recíproca dado que la primera sólo puede darse a través de la apropiación distintiva de ciertos repertorios culturales que se encuentran, ya sea en nuestro entorno social, en nuestro grupo o en nuestra sociedad. De este modo, la identidad es posible en razón de contener referentes culturales concretos compartidos por un grupo o por una comunidad dada, a partir de lo cual se llegan a establecer procesos de reconocimiento y diferenciación entre distintos actores sociales.
} 
Desde este ángulo de análisis, la escuela constituye un espacio multiforme a través de la cual se agregan formas singulares de representarse lo educativo. Esta circunstancia no sólo es producto de una actividad autorreferencial, propia de las condiciones escolares de operación regular, sino que es influenciada por significaciones de carácter global. La escuela actúa como una instancia de mediación cultural entre los significados, sentimientos y conductas de la comunidad social y el desarrollo de las nuevas generaciones. De ahí la conveniencia de recuperar un "<<enfoque ecológico>> de la escuela; es decir, una aproximación orientada a atender el cruce de culturas presente en estas instancias de socialización como resultado de la diversidad de producciones e intercambios simbólicos que vinculan la vida escolar con la vida social" (Pérez, 2004, p. 17). Son estas transacciones con la esfera sociocultural lo que propicia la presencia de variadas construcciones de sentido dentro de los procesos educativos.

Acorde con este presupuesto, se considera que la multiplicidad de los intercambios hace de los centros escolares lugares donde se combinan distintas actividades ordinarias, las cuales no se desenvuelven de manera desordenada; por el contrario, se articulan con normas, reglas, presunciones básicas, costumbres, que las condicionan tanto en su naturaleza como en su ordenamiento secuencial (Maslowski, 2001). La jornada escolar se vuelve, de este modo, en un conglomerado de prácticas y significaciones sobre el cual se organizan las relaciones sociales que estructuran lo cotidiano al interior de los establecimientos. Es esto lo que viene a representar su trama cultural; esto es, la producción de una particular forma simbólica de vivencia cotidiana signada por una implicación laboral y educativa.

Con base en este principio, la importancia de atender los componentes culturales asociados con la gestión escolar responde a la necesidad de avanzar en la comprensión de cómo son y cómo operan realmente las escuelas. Asunto no menor, por cuanto las expectativas de desarrollo de los diferentes países están enfocadas, en buena medida, en la revaloración de la educación como un elemento clave de progreso ante la creciente diversificación económica, la competitividad productiva a escala global, la amplitud de la interculturalidad así como la resignificación de la ciudadanía, entre otros aspectos. Ello exige volver la mirada a la unidad básica donde se concentran cotidianamente los esfuerzos para forjar a las nuevas generaciones; es decir, la escuela. En esta última, existen factores que muestran la intensidad y relevancia de los procesos culturales; entre ellos se pueden citar los siguientes:

- Es en las escuelas donde se pone en operación el currículum; un dispositivo sobre el cual se condensa una concepción particular de conocimiento, deudora de las circunstancias históricas que estructuran y le dan sentido a su presencia al interior de una sociedad en específico. En torno suyo se entabla una relación pedagógica entre un educador y un educando dentro de un ambiente de trabajo que reclama ciertas actitudes o disposiciones para lograr determinados propósitos educativos. 
- Para lograr las finalidades de la educación, la escuela requiere de una intervención colectiva ordenada con base en criterios de asignación y distribución jerárquica del trabajo. Se requiere, por tanto, de un procedimiento que le otorgue certeza a la actuación de los distintos agentes involucrados.

- La escuela organiza los usos del tiempo y del espacio a través de la existencia de horarios, ciclos, disposición espacial de las aulas, salas y talleres, segmentación de los grados, etc. Esta ordenación regula y estandariza las distintas actividades vinculadas a los procedimientos de socialización escolar, circunstancia impregnada de esquemas interpretativos por medio de los cuales cada agente le otorga sentido a su actuar.

- Al interior de la escuela se crean y reproducen rituales de socialización asociados al reconocimiento de la autoridad docente por parte de los alumnos, a la identificación de lo nacional o lo comunitario, a la celebración de festividades, al uso particular de un uniforme, al apego didáctico a los libros de texto, entre otros procesos. Al amparo de estas construcciones simbólicas se estructura una idea de enseñanza y, de forma complementaria, un sentido de la formación.

- La escuela es una instancia reguladora y certificadora de los aprendizajes como parte de una construcción sociohistórica. Es bajo el reconocimiento de esta autoridad simbólica como se inscriben imaginarios vinculados con expectativas, anhelos y esperanzas que la sociedad fija en los alumnos para proyectar un tipo de comportamiento futuro.

Es a través de estos y otros componentes como los centros construyen un conjunto de prácticas culturales alrededor de las finalidades educativas. La cultura escolar, por tanto, es un factor central de atención para indagar los diferentes comportamientos asociados a los imperativos actualmente en curso referidos a la calidad, la innovación, la eficiencia; los cuales repercuten en la definición de ser"escuela"y, por extensión, de quienes tienen la responsabilidad de llevar a cabo los compromisos emergentes de la educación.

A ese respecto, creemos importante delinear una posible agenda de investigación que aborde los siguientes puntos: i) las definiciones culturales asociadas a los estímulos de carácter meritocrático para la profesionalización y actualización de los docentes; ii) los significados asumidos en torno al liderazgo y sus efectos concurrentes en los procesos escolares; iii) las construcciones de sentido alrededor de la calidad educativa entre los participantes de la organización; iv) las interpretaciones vinculadas con los mecanismos de evaluación docente; v) los efectos de las pruebas estandarizadas en el ordenamiento cotidiano de la práctica profesional; vi) las diferenciaciones de género relacionadas con el ejercicio directivo; vii) las posibilidades de la inclusión educativa en escenarios de alta marginación y diversidad cultural; y vii) las formas de vinculación entre lo escolar y lo comunitario, especialmente en contextos marcados por la violencia social. 


\section{A manera de conclusión}

A partir de estas reflexiones se ha hecho un intento por destacar la relevancia analítica de centrar el estudio de la gestión desde un referente cultural. Las propuestas de reordenación de la gestión escolar invitan a en la indagación de los procesos educativos y sus formas de organización correspondiente. Ante la complejidad que rodea el funcionamiento de las escuelas, consideramos necesario revalidar la pertinencia de ubicar a los centros como la unidad de análisis fundamental para estar en posibilidad de comprender in situ los alcances efectivos de las nuevas exigencias condensadas en las políticas educativas, atrayendo para este efecto la dimensión simbólica-cultural.

Desde este ángulo, la gestión presenta una íntima vinculación con la cultura escolar, y ambos dominios los podemos ubicar desde tres referentes analíticos:

- Como parte de una construcción socio-histórica a través de la cual se producen, se recrean y transmiten contenidos de significación expresados en códigos, rituales, artefactos, creencias, valores y prácticas que ordenan y guían la acción educativa al interior de los centros.

- Como elementos que movilizan modos de apropiación específicos entre sus distintos agentes para darle sentido a sus orientaciones cotidianas donde se regulan los procesos de socialización escolar.

- Como componentes de significación que incursionan activamente al interior de las relaciones sociales a través de las cuales se produce una heterogeneidad de los intereses, convenciones, supuestos, valores o creencias acerca de la educación y sus implicaciones prácticas.

Estos componentes parten de una perspectiva simbólica, centrada en la interpretación de los significados en cuanto una construcción desarrollada por los sujetos en contextos sociales específicos. Esto supone ubicar la naturaleza de las prácticas escolares no únicamente como una expresión de racionalidad instrumental basada en el cálculo, o simplemente como una mera adaptación funcional a los imperativos normativos desprendidos de la institucionalidad educativa; sino como parte de un esquema de significación más amplio y alimentado por referentes de orden histórico-cultural por medio de los cuales se confronta, se conviene o se negocia la idea de "ser escuela" $y$, por extensión, la de "ser directivo" "ser maestro" o "ser alumno".

\section{Referencias}

Aguerrondo, I. (1993). La calidad de educación: ejes para su definición y evaluación. Organización de Estados Iberoamericanos para la Educación, la Ciencia y la Cultura (UNESCO). Recuperado en: http://www.oei.es/calidad2/aguerrondo.htm 
Candau, J. (2002). Antropología de la memoria. Buenos Aires, Argentina: Nueva Visión.

Díaz B., A. (2006). El enfoque de competencias en educación ¿Una alternativa o un disfraz de cambio? Perfiles Educativos, 28(111), pp. 7-36. Recuperado en: http://www.scielo.org.mx/ scielo.php?pid=S0185-26982006000100002\&script=sci_arttext

Giménez, G. (2009). Cultura, identidad y memoria. Materiales para una sociología de los procesos culturales en las franjas fronterizas. Frontera Norte, 21(41), enero-junio, pp. 7-32. México: COLEF.

Halbwachs, M. (1968). La mémorie collective. París: PUF.

Hargreaves, A. y Fink, D. (2008). El liderazgo sostenible. Siete principios para el liderazgo en centros educativos innovadores. Madrid, España: Morata.

Krauss, D. (1999). Appropriation et pratiques de la lecture. Les fondements méthodologiques et théoriques de l'approche de l'histoire culturelle de Roger Chartier. Laberynthe. Recuperado en: http://laberynthe.revues.org/56.

Maslowski, R. (2001). School culture and school performance. Netherlands: Twente University Press. Recuperado en: www.tup.utwente.nl/uk/catalogue/educational/school-culture.

Pérez G., A. (2004). La cultura escolar en la sociedad neoliberal. Madrid, España: Morata.

Rivas, J. I. (2003). La perspectiva cultural de la organización escolar: marco institucional y comportamiento individual. Educar, (31), pp. 109-119. Recuperado en: http://dialnet.unirioja. $\underline{\text { es/servlet/articulo?codigo }=643427}$

Rockwell, E. (2000). Tres planos para el estudio de las culturas escolares: el desarrollo humano desde una perspectiva histórico-cultural. Interacciones 5(09), pp. 11-25. Recuperado en: http://www.redalyc.org/articulo.oa?id=35450902

Sacristán, J. G. (1999). Poderes inestables en educación. Madrid, España: Morata. 